03,09

\title{
Интерференционное усиление конверсии поляризации света от структур с квантовой ямой
}

\author{
(ㄱ Л.В. Котова ${ }^{1,2}$, А.В. Платонов ${ }^{1}$, В.П. Кочерешко ${ }^{1}$, С.В. Сорокин ${ }^{1}$, С.В. Иванов ${ }^{1}$, Л.Е. Голуб ${ }^{1}$ \\ ${ }^{1}$ Физико-технический институт им. А.Ф. Иофрфе РАН, \\ Санкт-Петербург, Россия \\ ${ }^{2}$ Санкт-Петербургский национальный исследовательский университет \\ инфрормационных технологий, механики и оптики, \\ Санкт-Петербург, Россия \\ E-mail: Kotova@mail.ioffe.ru
}

\begin{abstract}
Оптическая активность кристаллов приводит к возникновению эллиптичности при отражении света, линейно поляризованного в плоскости интерфейса или нормально к ней. В исследованных структурах с квантовыми ямами, обнаруженный экспериментально эффект обусловлен спин-орбитальным взаимодействием и двулучепреломлением. Выявлена качественная зависимость эффекта от изменения ориентации линейной поляризации падающего света. Для света, поляризованного в плоскости падения, обнаружено резкое увеличение степени конверсии поляризации в узком диапазоне углов падения. Последнее связано с влиянием интерференции отраженной волны на толщине структуры.
\end{abstract}

Авторы благодарят за финансовую поддержку Российский научный фонд (проект № 16-12-10503). Работа В.П.К. была поддержана грантом РФФИ (15-02-04527).

DOI: 10.21883/FTT.2017.11.45052.22k

Спектроскопия экситонного отражения с поляризационным разрешением простой, с точки зрения аппаратной реализации, но эффективный метод исследования свойств полупроводниковых гетероструктур. Такой метод позволяет исследовать тонкие оптические эффекты и определять связанные с ними зонные параметры. В частности, этим методом был обнаружен эффект гигантского увеличения $g$-фактора экситона при его движении [1] и определены параметры гамильтониана дырки [2], ответственные за возникновение этого эффекта. В работе [3] из анализа спектров экситонного отражения в магнитном поле был выявлен специфичный для квантовых ям эффект магнитоиндуцированной пространственной дисперсии - эффект четности. В [4] была исследована зависимость величины диамагнитного сдвига экситона от волнового вектора его центра масс в поперечном магнитом поле. В настоящей работе данный экспериментальных подход использован для исследования явления оптической активности в квантовых ямах, связанной со спин-орбитальным взаимодействием в экситоне.

При изучении эффекта оптической активности в геометрии на отражение в эксперименте определяются параметры Стокса отраженного света для падающего света, поляризованного в плоскости падения ( $P$-поляризация) или нормально к ней ( $S$-поляризация) [5]. Параметры Стокса определяются обычным образом:

$$
P_{\text {circ }}=\frac{I_{\sigma^{+}}-I_{\sigma^{-}}}{I_{\sigma^{+}}+I_{\sigma^{-}}}, \quad \tilde{P}_{\text {lin }}=\frac{\tilde{I}_{1}-\tilde{I}_{2}}{\tilde{I}_{1}+\tilde{I}_{2}},
$$

здесь $I_{\sigma^{ \pm}}$- интенсивность света, отраженного в циркулярных компонентах, а $\tilde{I}_{1,2}-$ интенсивность линейно поляризованных компонент в осях, повернутых на $45^{\circ}$ относительно плоскости падения.
Ранее, в нашей работе [5] исследовался свет, падающий на образец в $S$-поляризации для всех углов падения. Было установлено, что при падении на образец света, линейно поляризованного в $S$-компоненте, отраженный свет оказывается, в общем случае, эллиптически поляризованным, то есть $P_{\text {circ }}$ и $\tilde{P}_{\text {lin }}$ отличны от нуля. Возникновение эллиптичности отраженного сигнала для линейно поляризованного падающего света естественно определить как конверсию поляризации.

Анализ этой угловой зависимости для $P_{\text {circ }}$ и $\tilde{P}_{\text {lin }}$, проведенной в [5], позволил нам установить, что конверсия поляризации связана с двумя вкладами. Первый вклад проявляется при нормальном падении света и вызван обычным двулучепреломлением, присущем самой структуре. Второй вклад возникает лишь при наклонном падении, а его величина пропорциональна волновому вектору света в плоскости ямы. Это явление оптической активности вызвано спин-орбитальным взаимодействием в экситоне, а именно вкладом Дрессельхауза. Эффект проявляется только на легком экситоне, так как необходимо наличие компоненты оптически активного диполя вдоль оси роста структуры.

В работе [5] величина конверсии поляризации не превышала $2 \%$ для $S$-поляризованного падающего света. В настоящей работе мы рассмотрим вопрос о возможности увеличения величины наблюдаемой поляризации, используя $P$-поляризованный падающий свет, а также эффект интерференции света в структуре.

Использование именно $P$-поляризованного света при исследовании спектров отражения от полупроводниковых структур с квантовыми ямами является традиционным подходом [6], позволяющим увеличить относительный вклад в отражение, связанный с КЯ. Действительно, 
для любой структуры с КЯ наблюдаемый сигнал отражения содержит в себе вклад не только от КЯ, но и от поверхности образца, а также, в некоторых случаях, и от границы структура-подложка. Для структуры с одиночной КЯ коэффициент отражения может быть представлен в виде [7]

$$
R=\left|r_{01}+r_{q w} e^{i \Phi_{1}}+r_{12} e^{i \Phi_{2}}\right|^{2}
$$

Здесь $r_{01}, r_{q w}, r_{12}$ - амплитудные коэффициенты отражения от поверхности, КЯ и подложки соответственно, $\Phi_{i}$ - набег фаз света в структуре. В (2) мы пренебрегли многократным отражением в структуре.

Последний вклад $\left(r_{12}\right)$ существен, например, в случае структур на основе $\mathrm{A}^{\mathrm{II}} \mathrm{B}^{\mathrm{VI}}$ полупроводников, выращенных на подложке GaAs, поскольку в этом случае разница показателей преломления между материалом структуры и подложки соизмерима с ней на границе воздухструктура.

При нормальном падении света, а также в $S$-поляризации амплитуда фонового сигнала $r_{b g}=\left|r_{01}+r_{12} e^{i \Phi_{2}}\right|$, как правило, существенно превосходит вклад от КЯ $\left(r_{q w}\right)$. Тем самым, наблюдаемый в спектре резонанс связан с членом, пропорциональным $r_{b g}^{*} r_{q w}$ в (2), а членом $\left|r_{q w}\right|^{2}$ можно пренебречь. Для слабых резонансов в случае $\left|r_{q w}\right| \ll\left|r_{b g}\right|$, это приводит к сложности интерпретации спектров отражения. Ситуацию можно улучшить, используя $P$-поляризацию падающего света. При наклонном падении $P$-поляризованного света сигнал, связанный с отражением от поверхности, монотонно уменьшается вплоть до угла Брюстера, для которого отражение от поверхности достигает нуля. Если отражение от подложки мало, то вблизи угла Брюстера можно реализовать ситуацию, когда фоновый сигнал и сигнал от КЯ соизмеримы [7]. В противоположном случае бо́льшего отражения от подложки фоновый $\left(r_{b g}\right)$, то есть не связанный с экситоном в КЯ, сигнал включает сумму амплитуд отраженного света от поверхности и от подложки с учетом фазы, набираемой светом при прохождении структуры. Таким образом, измеряемый фоновый сигнал есть результат интерференции двух вкладов, существенно зависящий от толщины структуры. Варьируя толщину, можно добиться как увеличения, так и подавления фонового сигнала.

Рассмотрим вышесказанное на примере исследованной структуры, схема которой представлена на рис. 1. Структура представляет собой одиночную КЯ $\mathrm{ZnSe} / \mathrm{Zn}_{0.82} \mathrm{Mg}_{0.18} \mathrm{~S}_{0.18} \mathrm{Se}_{0.82}$ шириной $10 \mathrm{~nm}$, выращенную вдоль направления [001] на подложке GaAs методом MBE (подробнее в [5]). Толщины барьеров справа и слева одинаковые, а общая толщина структуры составляет $230 \mathrm{~nm}$ (барьеры - $110 \mathrm{~nm}$ ), что соответствует, примерно, $5 \lambda / 4$, где $\lambda$ - это длина волны света на частоте экситона в $\mathrm{ZnSe}$. Тем самым, полный набег фазы $\Phi_{2}$ света, отраженного от подложки, будет равен 5л. Для такой разности фаз происходит деструктивная интерференция, и фоновый сигнал подавляется. Отметим здесь, что фаза $\Phi_{2}$ зависит от угла падения. Она уменьшается с увеличением угла. Оценим величину модуляции фоно-

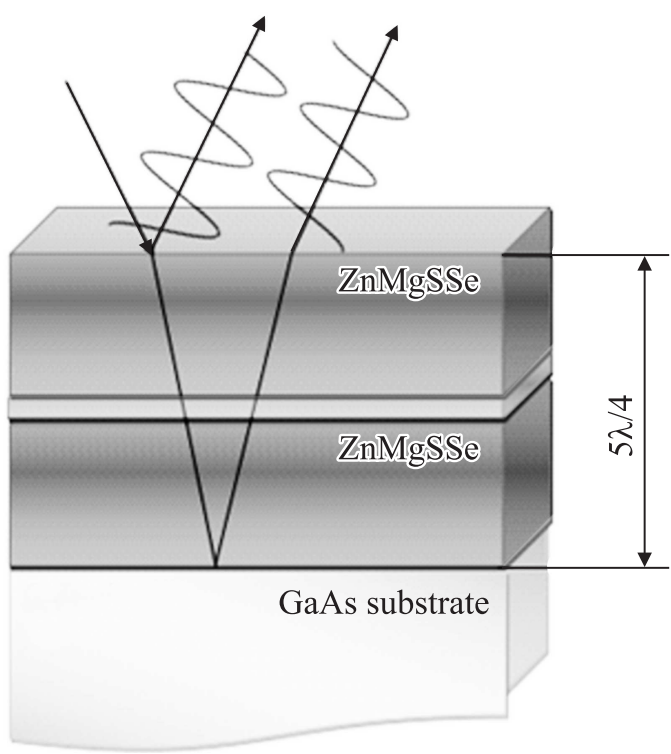

Рис. 1. Схематическая иллюстрация интерференционного усиления эффекта конверсии поляризации света при отражении от исследуемого образца. Ширины барьеров и КЯ данного образца составляют $110 \mathrm{~nm}$ и $10 \mathrm{~nm}$ соответственно, и их суммарная толщина равна $5 \lambda / 4$, где $\lambda$ - длина волны света на частоте экситона в $\mathrm{ZnSe}$. Это позволяет достигнуть полной компенсации отражения от покрывающего слоя и подложки, что приводит к возрастанию экситонного вклада в отражении.

вого сигнала с учетом реальных значений показателей преломления структуры $n=2.45$ и подложки $n=4.6$ при нормальном падении. В этом случае будем иметь $r_{01} \approx 0.4$ и $r_{12} \approx 0.3$. Видно, что для нормального падения фоновое отражение меняется в зависимости от фазы в диапазоне от $\left|r_{01}+r_{12}\right|=0.7$ до $\left|r_{01}-r_{12}\right|=0.1$, но не обнуляется. Однако при наклонном падении в $P$-поляризации ситуация меняется. Коэффициент отражения от поверхности $r_{01}$ уменьшается, в соответствии с формулами Френеля, а коэффициент отражения от подложки $r_{12}$ остается практически неизменным, так как угол падения света на подложку много меныше внешнего угла и никогда не превосходит $26^{\circ}$ в данной структуре. Для угла падения около $45^{\circ}$ вершины $r_{01}, r_{12}$ сравниваются. В тоже время, благодаря подобранному дизайну структуры, именно для этого угла фаза $\Phi_{2}$ равна $5 \pi$ для частоты света, соответствующей легкому экситону. В результате происходит полная компенсация фонового сигнала отражения $r_{b g} \approx 0$.

Экспериментальное проявление этого эффекта проиллюстрировано на рис. 2, где приведены два спектра отражения для углов падения $45^{\circ}$ и $35^{\circ}$. На спектрах хорошо видны два резонанса, соответствующих экситонным переходам с легкой и тяжелой дыркой. Видно, что вне резонансов фоновый сигнал испытывает сильную модуляцию в соответствии со сказанным выше. Минимум фонового сигнала находится в области экситонных переходов и смещается, как и должно быть, в красную область спектра с ростом угла падения. На рис. 2 можно 

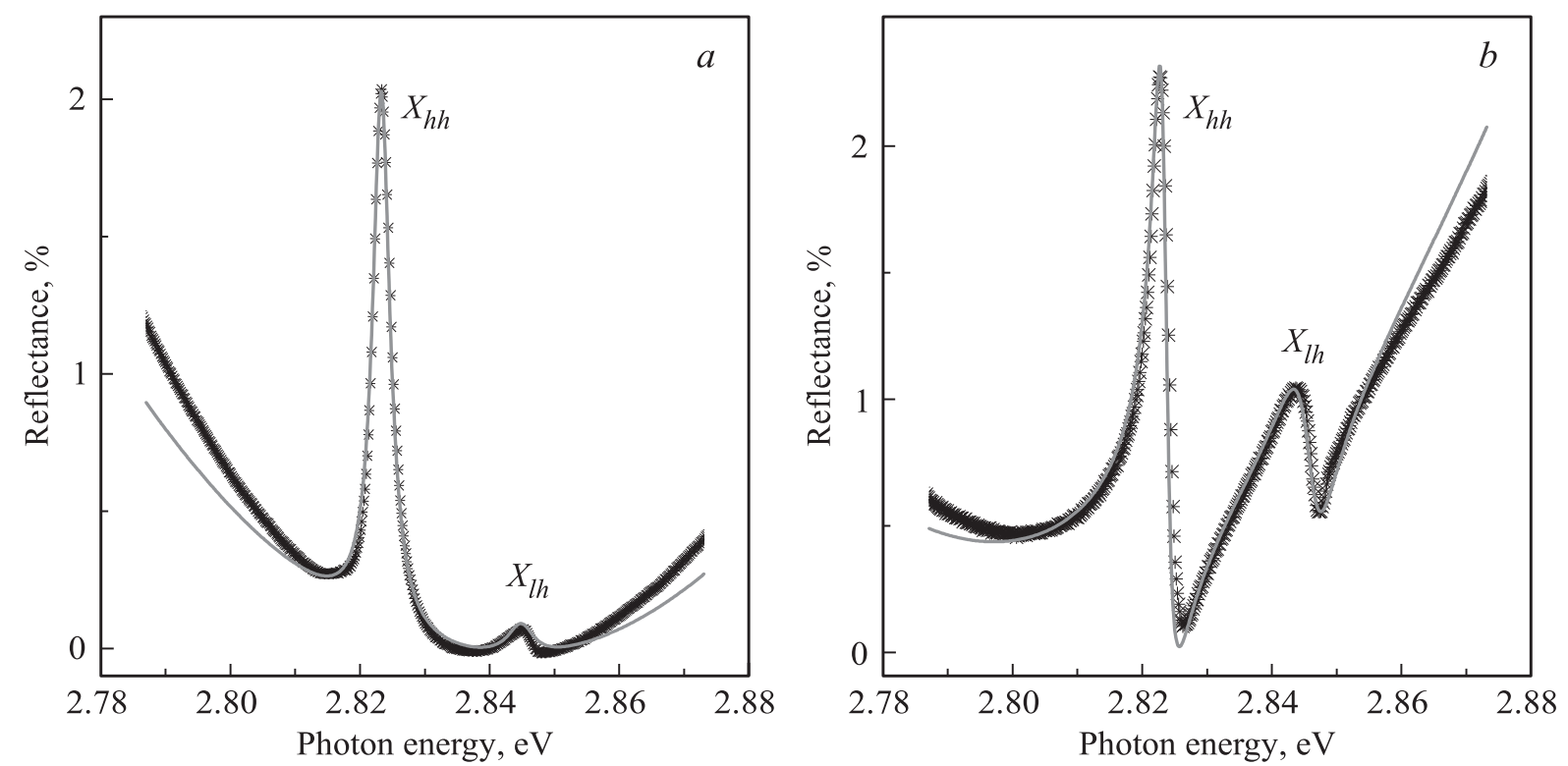

Рис. 2. Спектры отражения от структуры, измеренные при падении $P$-поляризованного света под углом $\theta=45^{\circ}(a)$ и $\theta=35^{\circ}(b)$. Точки - экспериментальные данные, сплошные кривые - теоретические зависимости. Указаны резонансы для легкого $X_{l h}$ и тяжелого $X_{h h}$ экситонов.

проследить, как при увеличении угла падения через минимум фонового отражения проходят резонансы сначала тяжелого экситона $X_{h h}$ (рис. $\left.2, b\right)$, а затем при $\theta=45^{\circ}-$ резонанс легкого экситона $X_{l h}$. Для угла $35^{\circ}$ значение $\left|r_{b g}\right|^{2}$ еще отлично от нуля, а для $45^{\circ}$ оно в точности равно нулю. И именно в этой точке спектра находится легкий экситон. Отметим еще раз, что такое совпадение является замечательным свойством именно выбранной структуры.

Проведя подгонку экспериментальных данных с использованием приближения, в котором резонансное поведение экситона в отражении описывается полюсной функцией [7] с характерными радиационным $\Gamma_{0}$ и нерадиационным затуханиями $\Gamma$, мы установили для легкого экситона $\hbar \Gamma_{0}=0.05 \mathrm{meV}$ и $\hbar \Gamma=2.35 \mathrm{meV}$ соответственно. Поскольку при $r_{b g} \approx 0$ наблюдаемый сигнал отражения в области легкого экситона связан только с резонансным вкладом $\left|r_{q w}\right|^{2}$ в формуле (2), его можно оценить как $\left(\Gamma_{0} / \Gamma\right)^{2}$. Это дает для амплитуды отражения в области легкого экситона при угле $45^{\circ}$ значение $R=\left|r_{q w}\right|^{2}=4.5 \cdot 10^{-3}$, что хорошо согласуется с рис. $2, b$.

Эффект полной компенсации фонового отражения носит резонансный характер в зависимости от угла падения. Проанализировав спектры отражения для различных углов падения $P$-поляризованного света от $0^{\circ}$ до $75^{\circ}$, мы получили зависимость отношения амплитуд отражения легкого экситона к амплитуде тяжелого на резонансной частоте, которая построена на рис. 3. Эта величина постоянна до $30^{\circ}$ и равна 0.25 , в соответствии с известным отношением оптических матричных элементов. Затем наблюдается яркий минимум для $\theta=45^{\circ}$, и после $55^{\circ}$ происходит возрастание опять до значения 0.25 . Особо следует отметить, что минимум резкий, то есть уменьшение амплитуды легкого экситона вплоть до его исчезновения из спектра происходит в узком угловом диапазоне. На рис. 3, также построена расчетная зависимость для отношения амплитуд, которая в точности повторяет экспериментальные данные. Последнее однозначно указывает на адекватность предлагаемой модели.

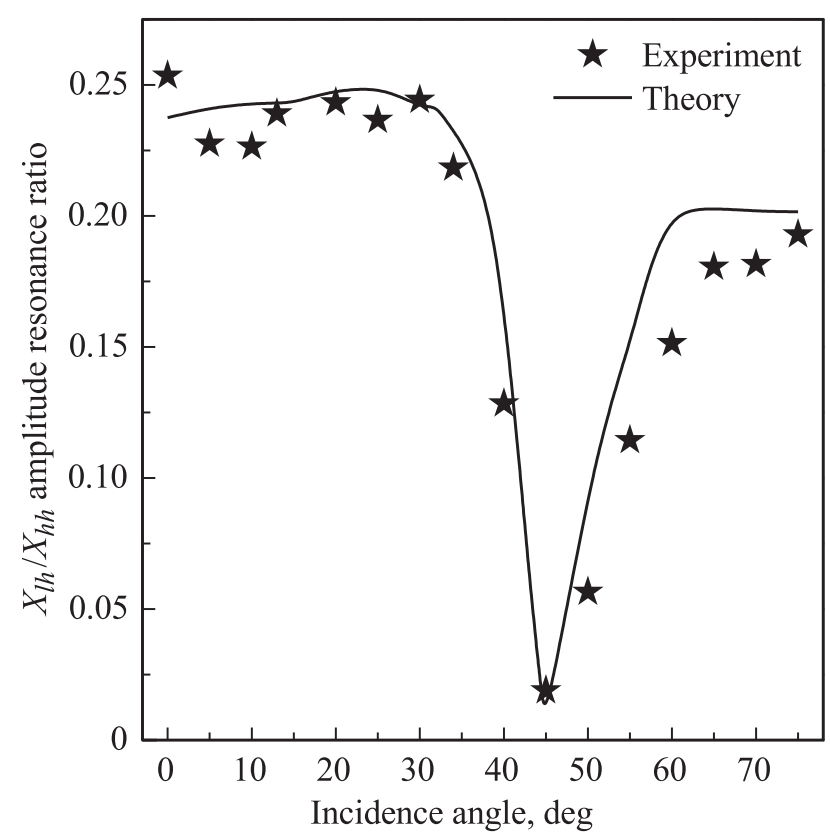

Рис. 3. Зависимость отношения амплитуды легкого экситона к амплитуде тяжелого на резонансной частоте экситона от угла падения $P$-поляризованного света. Символы - данные эксперимента, сплошная кривая - теоретически рассчитанная зависимость 

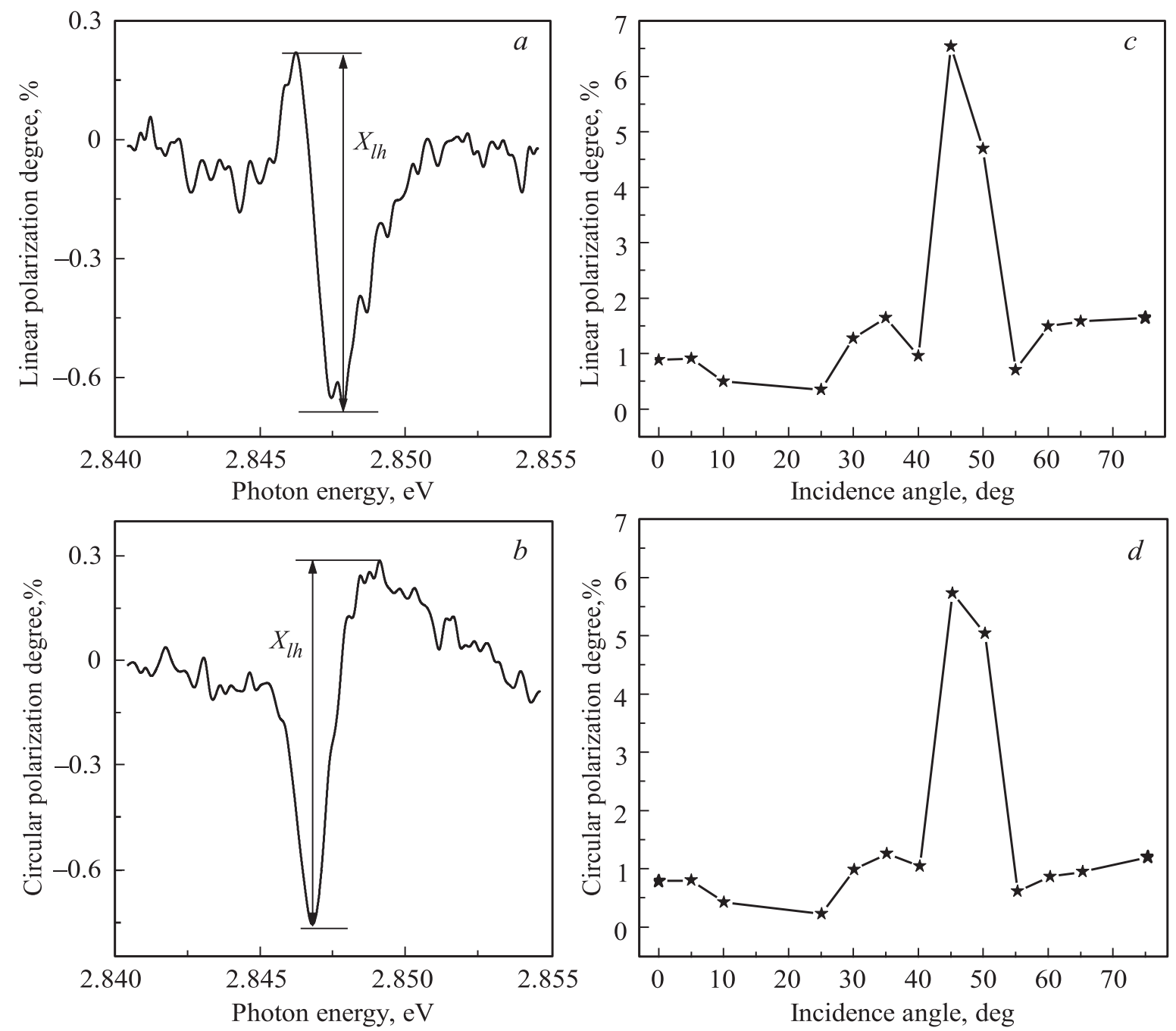

Рис. 4. Спектры поляризации отраженного света при наклонном падении под углом $\theta=25^{\circ}$ в области $X_{l h}$ резонанса для легкого экситона: $a)$ - степень линейной поляризации в осях, повернутых на $45^{\circ}$ относительно плоскости падения, $\left.b\right)-$ степень циркулярной поляризации. На основе измерения величин сигнала, полученных для различных углов падения $P$-поляризованного света аналогично указанным на рисунках $a, b$, представлены экспериментальные зависимости амплитуд для $(c)$ линейной под $45^{\circ}$ и $(d)$ - циркулярной степеней поляризации от угла падения света.

Перейдем теперь к обсуждению поляризационных свойств коэффициента отражения и проанализируем, как наблюдаемый на легком экситоне интерференционный эффект подавления фона влияет на измеряемую величину поляризации отражения, вызванную эффектом конверсии.

В самом общем случае эффект конверсии поляризации феноменологически может быть описан как [8]

$$
\left(\begin{array}{l}
E_{s}^{r} \\
E_{p}^{r}
\end{array}\right)=\left(\begin{array}{ll}
r_{s s} & r_{p s} \\
r_{p s} & r_{p p}
\end{array}\right)\left(\begin{array}{l}
E_{s}^{i} \\
E_{p}^{i}
\end{array}\right),
$$

здесь $E_{s, p}^{i}$ и $E_{s, p}^{r}-$ амплитуды падающей и отраженной волны, $r_{s s}$ и $r_{p p}-$ коэффициенты отражения, сохраняющие поляризацию, а $r_{p s}-$ член, ответственный за конверсию поляризации. На основании (3), несложно получить выражение для параметров Стокса, характе- ризующих величину конверсии. Для $P$-поляризованного падающего света они примут вид

$$
\begin{gathered}
P_{\text {circ }}=2 \operatorname{Im}\left(\frac{r_{p s} r_{p p}^{*}}{\left|r_{p s}\right|^{2}+\left|r_{p p}\right|^{2}}\right), \\
\tilde{P}_{\text {lin }}=2 \operatorname{Re}\left(\frac{r_{p s} r_{p p}^{*}}{\left|r_{p s}\right|^{2}+\left|r_{p p}\right|^{2}}\right) .
\end{gathered}
$$

И аналогично для $S$-поляризованного падающего света.

Для $S$-поляризованного падающего света коэффициент $r_{s s}$ всегда много больше, чем $r_{p s}$, и выражение для поляризации можно упростить

$$
P_{\text {circ }}=2 \operatorname{Im}\left(\frac{r_{p s}}{r_{s s}}\right), \quad \tilde{P}_{\text {lin }}=2 \operatorname{Re}\left(\frac{r_{p s}}{r_{s s}}\right) .
$$

С учетом значений величин $r_{s s}$ и $r_{p s}$, измеренных в [5], это дает величину в диапазоне $1-2 \%$ в зависимости от угла падения. 

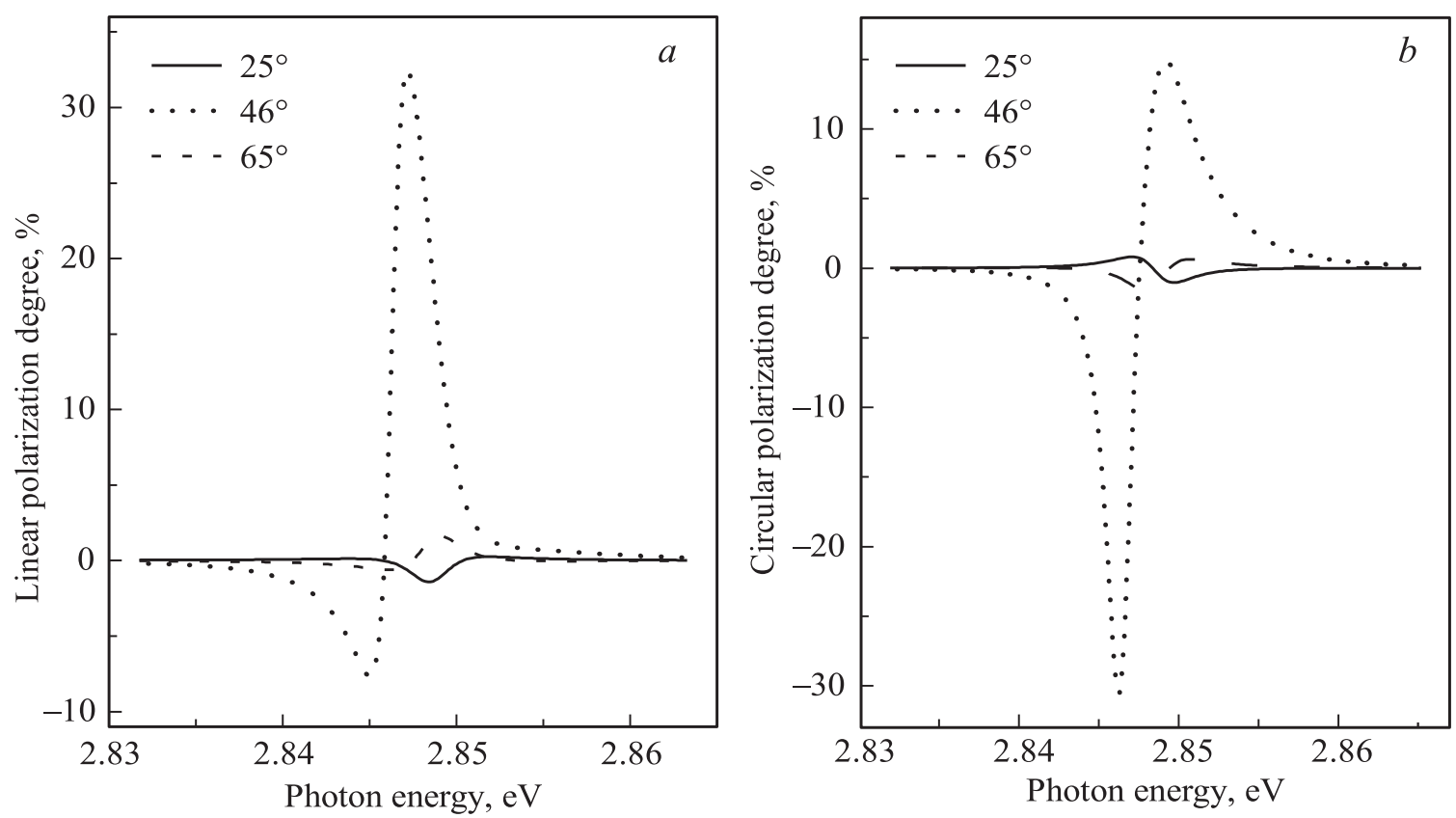

Рис. 5. Зависимости степеней линейной, повернутой на угол $45^{\circ}(a)$ и циркулярной $(b)$ поляризаций отраженного света, рассчитанные для трех углов падения света на исследуемый образец: $\theta=25^{\circ}$ (сплошная), $\theta=45^{\circ}$ (пунктирная), $\theta=65^{\circ}$ (штриховая).

Для $P$-поляризованного света, как было отмечено выше, возможна ситуация, когда коэффициент $r_{p p}=r_{q w}$, и он оказывается соизмерим с $r_{p s}$. В этом случае и спектральная, и угловая зависимости поляризации носят более сложный характер. Например, при $r_{p p}=0$, как следует из (4), циркулярная поляризация и линейная в осях, повернутых под $45^{\circ}$ к плоскости падения, отсутствует. В этом случае отраженный свет оказывается полностью линейно поляризованным перпендикулярно плоскости падения, а его интенсивность равна $\left|r_{p s}\right|^{2}$. Таким образом, происходит поворот плоскости поляризации на $90^{\circ}$. Разумеется, в реальной структуре сложно ожидать, что коэффициент отражения $r_{p p}$ исчезнет, в то время как коэффициент конверсии $r_{p s}$ останется конечным. Более вероятна ситуация, при которой эти величины близки. В этом случае возможна полная конверсия поляризации. При $r_{p p}=r_{p s}$ по модулю, поляризация достигает 100\%, то есть линейно поляризованный падающий свет отражается только в ортогональных Стоксовых компонентах.

В выбранной структуре нам удалось реализовать экспериментально подобные условия. На рис. 4 представлены данные по спектральным и угловым зависимостям параметров Стокса в области легкого экситона. На рис. 4, $a, b$ приведены спектральные зависимости $P_{\text {circ }}$ и $\tilde{P}_{\text {lin }}$ для угла падения $\theta=25^{\circ}$. После определения амплитуды степени поляризации, как показано на рис. $4, a, b$, на рис. $4, c, d$ была построена угловая зависимость этой величины для циркулярной и линейной компонент. Обе зависимости носят резонансный характер вблизи угла падения $45^{\circ}$, при этом максимальная величина конверсии поляризации достигает 6-7\%. Общая ширина максимума не превышает $5^{\circ}$, аналогично рис. 3. Данные, представленные на рис. 4, показывают, что $P$-поляризованный свет в совокупности с правильно подобранными параметрами структуры позволяет усилить наблюдаемый эффект конверсии поляризации отраженного света.

На рис. 5 построены теоретически рассчитанные спектральные зависимости параметров Стокса для набора углов $\left(\theta=25^{\circ}, 45^{\circ}, 65^{\circ}\right)$ с учетом реальных параметров, исследованной структуры. Общий характер этих зависимостей соответствует рис. 4 , однако величина конверсии для $\theta=45^{\circ}$ оказывается на порядок больше. Отличие расчетных и экспериментальных кривых легко объяснимо, если учесть конечную сходимость падающего пучка в эксперименте. Действительно, ширина максимума конверсии в угловой зависимости $5^{\circ}$, оказывается близкой к сходимости пучка $3^{\circ}$. Тем самым, в эксперименте происходит усреднение по углу, и степень конверсии оказывается заниженной по сравнению с достижимой для идеального параллельного луча падающего света.

В заключение, в работе был изучен эффект конверсии оптической поляризации света при отражении от структуры с квантовой ямой. Экспериментально определены параметры Стокса отраженного света для $S$ - и $P$-поляризаций, падающего в зависимости от угла падения. Установлено, что для в $P$-поляризации падающего света в угловой зависимости наблюдается узкий максимум, связанный с эффектом интерференционного подавления фонового отражения. Теоретическое моделирование показало, что для реальных параметров исследованной структуры степень конверсии может достигать десятков процентов в случае идеально параллельного пучка падающего света. 


\section{Список литературы}

[1] J.J. Davies, D. Wolverson, V.P. Kochereshko, A.V. Platonov, R.T. Cox, J. Cibert, H. Mariette, C. Bodin, C. Gourgon, E.V. Ubyivovk, Yu.P. Efimov, S.A. Eliseev. Phys. Rev. Lett. 97, 187403 (2006).

[2] L.C. Smith, J.J. Davies, D. Wolverson, H. Boukari, H. Mariette, V.P. Kochereshko, R.T. Phillips. Phys. Rev. B 83, 155206 (2011).

[3] В.П. Кочерешко, В.Н. Кац, А.В. Платонов. Изв. РАН. Сер. физ. 78, 12, 1649 (2014).

[4] Д.К. Логинов, В.П. Кочерешко, А.В. Платонов, J.J. Davies, D. Wolverson, L.C. Smith, R.T. Cox, J. Cibert, H. Mariette. ФTT 51, 1555 (2009).

[5] L.V. Kotova, A.V. Platonov, V.N. Kats, V.P. Kochereshko, S.V. Sorokin, S.V. Ivanov, L.E. Golub. Phys. Rev. B 94, 165309 (2016).

[6] E.L. Ivchenko, V.P. Kochereshko, I.N. Uraltsev, D.R. Yakovlev. Phys. Status Solidi B 161, 217 (1990).

[7] Е.Л. Ивченко, В.П. Кочерешко, А.В. Платонов, Д.Р. Яковлев, A. Waag, W. Ossau, G. Landwehr. ФТТ 39, 2072 (1997).

[8] Е.Л. Ивченко, А.В. Селькин. ЖЭТФ 49, 933 (1979). 